\title{
Excreción de Calicreína Urinaria en Niños Sanos a Distintas Edades
}

\author{
De. Carlos Saieh A. ${ }^{1}$; Dra. Gloria Valdes S. $^{2}$; T.M. Maricarmen Tobar $C^{2}{ }^{2}$ T.M. Cecilja Chacón L. ${ }^{2}$; \\ Dr. Hétor Croxato R. ${ }^{3}$
}

Urinary Kallikrein Excretion in Children

\begin{abstract}
Urinary Kallikrein excretion (UKE) was measured in 53 children aged 12 days to 15 ycars old (mean 6.73 years) coming from hospital wards or ombulatory clinics: all were free of renal patenchimatous diseases, arterial hypertension, or alterations of retual hemodinamic and none had recent history of drugs ingestion or parenteral tluids administration. The lower UKE. $(0.30 \pm 0.92 \mathrm{mU} 24 \mathrm{hts})$ were registered from infants under six months of age. Older infants and children showed UKE values rangin from $4.46 \pm 2.7$ and $16.1 \pm 3.7 \mathrm{mU} \times 24$ hrs. respectively. This results are apparently related to age differences in renal maturation.

(Key words: Age. Kalliksein urinary output. Renal maturation).
\end{abstract}

En 1926 Frey y col.' denominaron Calicreína (KAL) a una proteina que corresponde al primer componente del sistema que genera bradicinina, substancia hipotensora, vasodilatadora y con acción diurética y natriurética ${ }^{2} \cdot 4 \cdot 6 \cdot 8 \cdot 10-17$. Este sistema fia sido investigado en animales y en humanos. Actualmente se sabe que hay dos diferentes calicteínas, la glandular (renal) y la plasmática, diferentes desde el punto de vista estructural e inmunológico. La forma renal o glandular, es eliminada en gran parte por la orina y actúa como una hormona local ${ }^{2-19}$, está involucrada en la regulación del flujo sanguineo, la excreción del agua, los electrolitos y la síntesis de las prostaglandinas ${ }^{18}$. Rablero y $\mathrm{Col}^{20}$ experimentando con riñones perfundidos, detectaron pasaje de calicreina al líquido de perfusión $y$ : aunque los pulmones constituyen una excelente barrera química para las cininas, la calicteína propiamente tal no es afectada por el pulmón "in vivo"19 con lo que es posible postular que ejerza un papel vasodilatador sistérnico.

$\mathrm{Se}$ ha demostrado que este sistema tiene variaciones circadianas de la excreción, que también puede ser modificada por la aldosterona pero no por el flujo urinariols.

1. Unidad de Nefrología. Depto. de Pediatría, Div. Cs. Médicas Oriente, Facultad de Medicina, Hospital Luis Calvo Mackenna. Universidad de Chile.

2. Laboratorio de Nefrología. Depto. de Medicina. L'niversidad Católica de Chile.

3. Laboratorio de Fisiología. Facultad de Medicina. Universidad Católica de Chile.
El sistema calicreina-cinina tiene muchas similitudes con el sistema renina-angiotensina, aunque son antagónicos en una misma función. Ambos incluyen reacciones enzimáticas en cascada que culminan con la formación de péptidos muy potentes de propiedades opuestas: la angiotensina $\mathrm{II}$, vasocontrictor y retenedor de sodio, y la bradicinina con propiedades vasodilatadora, diurética y natriurética, comfartiendo ambos productos una enzima convertidora común (Fig. 1).

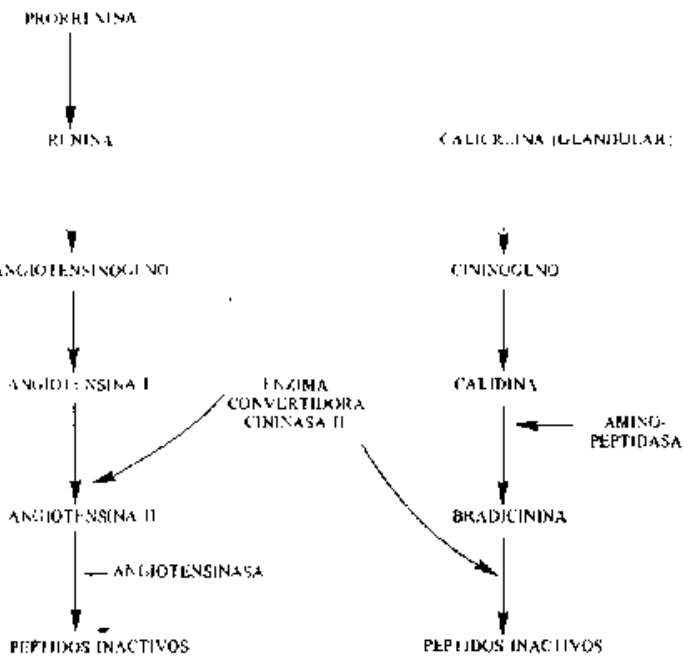

Figura 1: Esquema simplificado del metabolismo de Angiotensina Il y Bradicinina. 
El paralelismo fisiológico entre el sistema renina angiotensina y su antagonista calicreinabradicinina se ve acentuado por una respuesta similar a la escasez y a la sobrecarga de sodio $^{3-22}$. Las cininas a su vez son estimuladores de la producción de prostaglandinas, activando la prostaglandina-sintetasa, que transforma el ácido araquidónico en prostaglandinas, potenciándose así el efecto de las cininas.

La excreción de calicreína urinaria es significativamente menor en lactantes, preescolares $y$ niños hipertensos ${ }^{7-10}$ que en adultos. Además el sistema renina angiotensina posee gran actividad en los primeros meses de la vida ${ }^{1}$, lo que podria explicar la mayor resistencia vascular renal en los recién nacidos y lactantes menores, con respecto a escolares y adultos.

El estudio que presentamos se propone describir cuantitativamente la excreción de calicreina en la orina de distintos grupos etarios en nuestro medio.

\section{MATERIAL Y METODO}

Se estudiaron 53 niños de ambos sexos, 33 varones y 20 mujeres. cuya edad promedio fue de 6,73 años, con rango entre 12 días y 15 años. Se incluyeron pacientes hospitalizados y de la consulta ambulatoria del Hospital Luis Calvo Mackenna que, en el momento del estudio, no sufrian afecciones que comprometieran el parénquima ni ta hemodinámica renal, ni hubieran recibido drogas ni infusiones endovenosas en los 3 dias anteriores al estudio. En todos se hicieron por lo menos tres diferentes mediciones de la presión arterial, obteniéndose siempre valores en el rango normal.

Las muestras de orina fueron obtenidas mediante recolectores en los menores de dos años y directamente en los mayores, fueron mantenidas a $4^{\circ} \mathrm{C}$, mezcladas con Toluol, $0.10 \mathrm{ml} / \mathrm{kg}$ de peso corporal, filtradas a $20^{\circ} \mathrm{C}$ hasta el procesamiento.

Se midió diuresis diariamente. La excreción de sodio se determinó en la orina de 24 horas usando fotómetro de llama Coleman (IL) y la creatinuria sigujendo el método de Jaffe. La función renal se evaluó mediante la đepuración estimada de creatinina ${ }^{24}$.

La calicreina de midió con el método amidásico de Amundsen y col. ${ }^{23}$ con sustrato sintético $\mathrm{S}-2266$ y sus cifras se expresaron en micro-uni. dades por $24 \mathrm{hrs}$. (KAL 24) y por $100 \mathrm{gr}$. de tejido renal (KAL \%) (Figuras 2 y 3 ).

Para calcular KAL 1\% se estimó el peso en gramos de cada riñón en las distintas edades según las tablas empleadas en el Departamento de

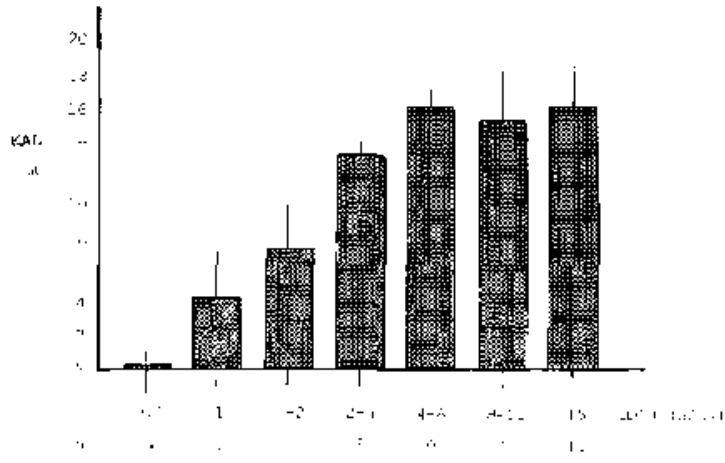

Figura 2: Calicreína (KAL) urinaria en 24 horas versus cdat.

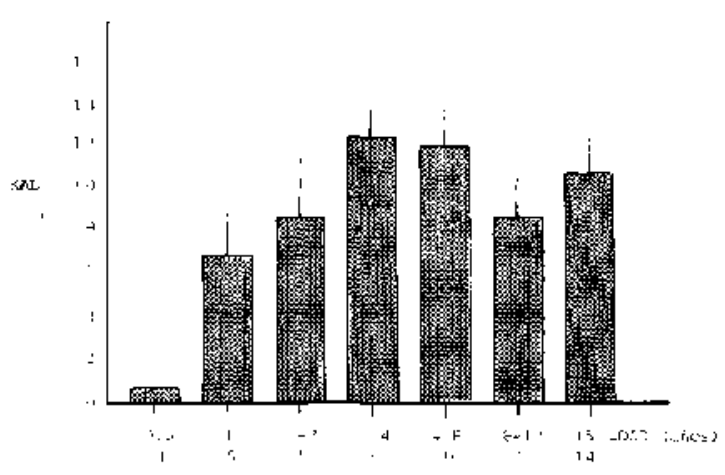

Figura 3: Caliereina (KAL) urinaria de 24 horas en rela ción a $100 \mathrm{gr}$. de tejido renal versus edaú.

Anatomía Patológica del Hospital Luis Calvo Mackenna.

Los resultados se expresan como promedios \pm el error estándar. Se consideró significativos un valor para $2 \mathrm{p}$ menor de 0,05 (Prueba de Student).

\section{RESULTADOS}

La calicreina urinaria en los menores de 6 meses expresada en valores absolutos relativos a la masa renal estimada es significativamente menor a la del niño mayor, siendo, las diferencias, significativas a partir del primer año de vida $(\alpha<$ 0.001 ).

Las concentraciones urinarias de Sodio son menores en los primeros 6 meses de edad $y$ a los 1 y 2 años de edad, observándose un aumento progresivo desde los 2 a los 15 años (Tabla 1).

El volumen de orina y la excreción de creatinina, aumentaron progresivamente con la edad. El índice de correlación entre excreción de calicreina $\mathrm{y}$ edad para el total fue $\mathrm{r}=0.40 \mathrm{y}$ aumentó a 0.60 al considerar a los niños menores de 2 años separados del resto. 
Tabla 1

Excreción de Calicrcína (KAL) en Niños Menores

\begin{tabular}{|c|c|c|c|c|c|c|c|}
\hline \multirow[b]{3}{*}{ KAL $24 \mu \mathrm{U}$} & \multicolumn{4}{|c|}{ Meses } & \multicolumn{2}{|l|}{ Años } & \multirow[b]{2}{*}{$12-15$} \\
\hline & $6 \pi$ & $6-12$ & $1-2$ & $2--4$ & $4-8$ & $8-12$ & \\
\hline & $0.3 \pm 0.29$ & $4.46 \pm 3$ & $7.4 \pm$ & $13 \pm 2$ & $16 \pm 3$ & $15 \pm$ & $16 \pm$ \\
\hline $\mathrm{K} \cdot \mathrm{AL} \% \mathrm{~mL}$ & $0.6 \pm 0.32$ & $4.46 \pm 3$ & $9 \pm$ & $13 \pm 2$ & $12 \pm 2$ & $9 \pm$ & $10 \pm$ \\
\hline Sod. Ur.m Eq/1 & $13 \pm$ & $68 \pm 35$ & $28 \pm$ & $\$ 5 \pm 8$ & $80 \pm 16$ & $99 \pm$ & $134 \pm 20$ \\
\hline Creat. Ur. mg $\%$ & $21 \pm 10$ & $58 \pm 10$ & $83 \pm 17$ & $206 \pm 49$ & $222 \pm 31$ & $407 \pm 102$ & $876 \pm 85$ \\
\hline Vol Ur. ml & $65 \pm 40$ & $256 \pm 61$ & $514 \pm 115$ & $572 \pm 91$ & $685 \pm 68$ & $951 \pm 98$ & $1126 \pm 131$ \\
\hline
\end{tabular}

\section{DISCUSION}

La excresión urinaria de calicreína es relativamente baja en nifios menores de 6 meses, expresada en microunidades por 24 horas o por $100 \mathrm{gr}$. de tejido renal. Lo que sugiere que, su síntesis está probablemente influenciada por el proceso de maduración, especialmente de los segmentos de conexión de los túbulos distales con los colectores, en los que se han descrito gránulos de calicreína 1 3-14-21.

A medida que avanza la edad, aumenta la excreción de calicreína $7-8-9-10-12$. De acuerdo con nuestros resultados los valores más bajos de calicreina se observan antes de los.6 meses de edad, luego éstos aumentan progresivamente para estabilizarse después de los dos años, sugiriendo estas cifras inmadurez en la síntesis de la proteina. Recientemente Valdés y col. han propuesto la existencia de un proceso de maduración de las células tubulares, con un rápido cambio morfofuncional, desde el punto de vista de la excreción de calicreína en orina, a los 6 meses pues antes de esa edad no se observaron grảnulos de calicreina (caligránulos) en cortes de riñones procesados mediante inmunocitoquímica para microscopía óptica ${ }^{16}$, lo que sig. nificaria que en ese grupo de edadès no habrian sitios anatómicos de elaboración de calicreina.

El significado cuantitativo de estos hallazgos aparece claro, no así el efecto fisiológico de la menor excreción de un principio vasodilatador durante los primeros meses de vida sobre la hemodinámica renal y sistémica: tanto en el recién nacido como el lactante la actividad de renina plasmática es elevada y la excreción de calicreina urinaria baja, con respecto a los niños mayores y adultos, lo que podría explicar en parte la relativamente mayor resistencia vascular y el menor flujo sanguíneo renal que se observa en los menores. Además del factor de inmadurez del túbulo, se puede postular que, la ausencia de un sistema renal con potente efecto natriurético y diurético, como es el sistema calicreína-bradicinina, juega un papel importante en el lactante menor, grupo etario especialmente lábil en relación al equilibrio hidrosalino.

Llama la atención que el descenso de un factor vasodilatador no se acompañe de hipertensión arterial sistémica en el recién nacido $y$ lactante, siendo muy probable que la ausencia de este sistema vasodilatador esté compensado por los niveles de prostaglandinas, fundamentalmente $E_{2}$ y $\mathbf{F}_{2}$ : Godard ${ }^{7}$ encontró que los valores de prostaglandinas en diferentes edades son muy semejantes (rango entre 500 a $800 \mathrm{mg} / \mathrm{m}^{2} / \mathrm{dia}$ para $F_{2}$ y 100 a 200 para $E_{2}$ ) en el recién nacido, lactantes $y$ adultos cuando se les corrige para la superficie corporal, lo que permite suponer que existe un equilibrio entre los sistemas vasodilatadores y vasocontrictores capaz de mantener una presión optima en cualquier edad.

\section{RESUMEN}

La calicreína es una proteína que genera. luego de varias transformaciones, bradicina, una sustancia con potente acción vasodilatadora.

En 53 niños de ambos sexos, sanos y normo. tensos, cuyas edades fluctuaron entre 12 dias y 15 años, se midió calicreína urinaria, expresándola en su valor absoluto y en relación a la masa renal. La calicreina urinaria es francamente inferior en el grupo de menores de 6 meses. ( $0.30 \pm 0.29$ uU 24 hrs.) con respecto a los valores obtenidos en los lactantes.y niños mayores $(9.46 \pm 2.7$ y $16.1 \pm 3,7 \mathrm{uU} 24 \mathrm{hrs}$. $)$.

Concluimos que la excreción urinaria de calicreína es significativamente menor en los menores de 6 meses, lo que puede estar relacionada con la maduración renal.

\section{REFERENCIAS}

1. Frey E.K., Krant $H$. and Wesle $E$.: Uber einen von der Niere ausgerschiedenen, die Herztatigkeit, anregenden Stoff. Hoppe. Seyler. S. Physiol. Chem. Phys. 157: 32, 1926. 
2. Croxatto H.R.: Calicreina renal y presión sanguinea. Rev. Med. Chil, 109: 143, 1981.

3. Wong P.Y., Takamo R.C. Williams H.G. Colman R.W: Response of Kallicrein kinin and renin angiotensin systems to saline infusion and upright posture. J, Clin.. Invest. 55: 691, 1975

4. Rozas R., Abertini $R$. and Coxatto $H$.: Renal Kallikrein and renal hypertensión. Mayo Clin. Proc. 52: $459,1977$.

5. Abe K., Sato $M$., Imai J. et al.: Renal kallicreinkinin: Its relation to renal prostagladios and renin-anglotensin-aldosterone in man. Kidney lnt 19869.1981 .

6. Klotman P.E., Trellis D.R., Coffman T.M.: Brady kinin stimulates oxygen comsumption and thromboxanc production in purified proximal tubule cell suspensions. Clin. Res. 31: 862 A, 1983.

7. Godard C., Yalloron H.B. and fave L.: Utinary prostaglandins vasopressin and kallicrein in healthy children from birth to adolescence. J . Pediatr. 100: $898,1982$.

8. Zimer S.H., Margolius H.S., Rosnar B. and Kass $\boldsymbol{E} . H_{1}:$ Stability of blood pressure rank and urinary kallikrein concentration in childhood: an ejght year follow-up. Circulation S8: $908,1978$.

9 Sinaika A,R., Glassen R.J., Gillum R.F. and Prineas $R . J .:$ Urinary kallikrein in excretion in grade school children with high and low blood pressure. I Pediatr. 100: 938, 1982 .

10. Naka T, Ogihara T., Hara $T$. et al.: The effect of aging on urinary kallikrein excretion in normotensive subjects and in patients with essential hyptrtensión J. Clin. Findocrinol. Metab. \$2: 1023, 1981.

11. Saieh C., Idatzoaga V., López G. et al.: Plasma renin activity in normal and malnourished children. Fur. J. Pediatr. 140: 184, 1983

12. Holland B. Clend J.M., Branstein H.: Urinary kallkrein excretion in essential and mineralocorticoid hypertension. J. Clin. Invest. 65: 347, 1980.

13. Carretero O.A., Scichi A.G.: Renal kallikrein its localization and possible role in renal function. Fed.
Proc. 35: 194. 1976.

14. Orstanik T.B., Nustad K., Bnandtzaeg P. and Pierce $J . Y$ " Celular origir of urinary kallikreins. J. Histochem. Cytochem. 24: 1037, 1976.

15. Carretero O.A., Sucti A.G.: The renal kallikrein-kinin system in human and in experimentel hypertension. Klin. Woichenschr. 56 (suppl. 1) 113,1978

16. Valdés $G$., Saieh $C$, Vio $C$. Salos $S$, Croxatto H.R. Chacón C. y Tobar M.C.: Evolución de la Calicreína renal a lo largo de la niñez. Reunión amual de la Sociedad de Biologia de Chile. F.1 Quisco, Nov., 1.984. Chile.

17. Vinci J.M., Zusman RE., Izzo J.J., Bowder R.E. Horwitz D., Pisano J.J. and Keiser $H R$.: Huntan urinary and plasma kinis. relatioships to sodium-retaining steroids and plasm renin activity. Curc. Res. 44: 228,1979

18. Croxarfo H. R. : Calicreína renal y presión arterial. Rey. Med. Chile. 109: 143. 198J.

19. Rosas $R_{+}$Palominos $G$, Croxatto H.R.: Mechanisms of action of renal kallikrein on renal pressure. The fifth stientific meeting of lnteramerican Socjety of Hypertenswon. March. 1983. Guaruja. Brazil.

20. Roblero $J$., Croxatto $H R .$, y Garcia $R$.: Kallikrein activity in perfustes and urine of isolated rat kidney. Am. J. Physiol. 231: 5, 1976.

22. O'Comor D.G.: Response of the renal Kallikreinkinin system intravasculat volume and tenal hemodynamics to sotium restriction and diuretic therapy. Hypertension (Supply III) 3: 72, 1982.

23. Amundsen E. Putter $J$., fribeger $P$, et al: Method for detcrmination of glandular kallakein by mean of a chromogenic triptide substrate. In: Kinins II, Biochemistry pathophisiology and clinical aspects, Ed. Fujii S. Moriya H., Juzuky T. Plenum Press, New York. 1979. p. 83

24. Saich C., Puga F., Froile E. y Rodriguez E.: Valoración de la función renal en la intancia por un método sencillo. Rev. Chil. Pediats. 49: 52, 1978. 